\title{
Boundary Effects on Eigen-problems of Discrete Laplacian in Lattices
}

\author{
Yueh-Cheng Kuo and Shih-Feng Shieh*
}

\begin{abstract}
We consider how distribution of eigenvalues depends on boundary conditions of a discrete Laplacian operator on lattices. We study the Laplacian with boundary conditions given by a linear combination of Dirichlet and Neumann conditions. In particular, we derive a secular equation and investigate the Laplacian operator's eigenvalues with different boundary conditions, including the interlacing property, the first eigenvalue gaps, and the monotonicity property.
\end{abstract}

\section{Introduction}

In this paper, we study the eigen-problems of the discrete Laplacian operator with boundary condition given by a linear combination of Dirichlet and Neumann conditions, i.e., Robin boundary condition. In 1D lattice, the negative discrete Laplacian matrix is given as follows:

$$
L \equiv L(\alpha, \beta)=\left[\begin{array}{ccccc}
2-\alpha & -1 & & & \\
-1 & 2 & -1 & & \\
& \ddots & \ddots & \ddots & \\
& & \ddots & \ddots & -1 \\
& & & -1 & 2-\beta
\end{array}\right] \in \mathbb{R}^{n \times n}
$$

where $\alpha, \beta \in \mathbb{R}$. Here, the parameters $\alpha$ and $\beta$ come from the boundary effects of differential equation: $-\phi^{\prime \prime}(x)=\lambda \phi(x)$ for $x \in[0,1]$ with boundary conditions

$$
c_{\ell} \phi^{\prime}(0)+d_{\ell} \phi(0)=0 \quad \text { and } \quad c_{r} \phi^{\prime}(1)+d_{r} \phi(1)=0
$$

Partition the interval $[0,1]$ into $n$ subintervals of uniform width $h=1 / n$ whose endpoints

$$
x_{k}=k h-h / 2 \quad \text { for each } k=0,1, \ldots, n+1 .
$$

Received July 19, 2021; Accepted February 11, 2022.

Communicated by Chiu-Yen Kao.

2020 Mathematics Subject Classification. 93B60, 34L15.

Key words and phrases. eigenvalue gaps, eigenvalues, discrete Laplacian.

*Corresponding author. 
Let $u_{j}$ approximate $\phi\left(x_{j}\right)$ and $\mathbf{u}=\left[u_{1}, u_{2}, \ldots, u_{n}\right]^{\top} \in \mathbb{R}^{n}$. The discretized version of the above boundary conditions is

$$
c_{\ell} \frac{u_{1}-u_{0}}{h}+d_{\ell} \frac{u_{0}+u_{1}}{2}=0 \quad \text { and } \quad c_{r} \frac{u_{n+1}-u_{n}}{h}+d_{r} \frac{u_{n}+u_{n+1}}{2}=0 .
$$

This implies that $u_{0}=\alpha u_{1}$ and $u_{n+1}=\beta u_{n}$, where

$$
\alpha=\frac{2 c_{\ell}+h d_{\ell}}{2 c_{\ell}-h d_{\ell}} \quad \text { and } \quad \beta=\frac{2 c_{r}-h d_{r}}{2 c_{r}+h d_{r}}
$$

provided that $2 c_{\ell}-h d_{\ell}$ and $2 c_{r}+h d_{r}$ are not zeros. Hence, $\frac{1}{h^{2}} L \mathbf{u}=\lambda \mathbf{u}$ is the eigenvalue problem of discrete Laplacian operator, where the matrix $L$ has the form in (1.1) and $\alpha$, $\beta$ are in 1.2 .

The eigenvector $\mathbf{u}=\left[u_{1}, u_{2}, \ldots, u_{n}\right]^{\top} \in \mathbb{R}^{n}$ of $L$ in (1.1) satisfies

$$
\begin{gathered}
-u_{k-1}+2 u_{k}-u_{k+1}=\lambda u_{k}, \quad k=1, \ldots, n, \\
u_{0}=\alpha u_{1}, \quad u_{n+1}=\beta u_{n},
\end{gathered}
$$

where $\alpha, \beta \in \mathbb{R}$ are given in 1.2 . Here, for $\alpha=\beta=0,(1.3 \mathrm{~b}$ represents a Dirichlet boundary condition, and for $\alpha=\beta=1,(1.3 \mathrm{~b})$ represents a Neumann boundary condition.

Robin boundary conditions are commonly used in solving Sturm-Liouville problems, which appear in many contexts in science and engineering, see 1, 4, 18. An open problem is proposed by Professor Shin-Tung Yao in the International Congress of Chinese Mathematicians 2019 [14 as follows:

Given a compact manifold $M$ with boundary, we can look at the Laplacian with boundary condition given by a linear combination of Dirichlet and Neumann conditions. Hence we have a way to join the Laplacian with Dirichlet condition to Laplacian with Neumann condition and back to Dirichlet condition. The eigenvalues and the eigenfunctions move accordingly. During this motion, we shall arrive at eigenvalues with multiplicity. Can one describe the dynamics of such a movement?

Such an eigen-problem has been investigated by many researchers. The first eigenvalue gaps for the Laplacian and discrete Laplacian operators have been investigated by $[3,5,9,16]$. Eigenvalue ratios have been studied by 2,8 . The number of eigenvalues trapped in the square-well potential has been studied in [10]. In paper [12], the eigenvalue problem of the discrete Laplacian on $\mathbb{Z}^{3}$ is studied. Such results are applied to the discrete Schrödinger operators on higher dimensional lattices. In [13], the distribution of eigenvalues of the discrete Schrödinger operators are studied. In [11], the expansion of eigenvalues of the discrete bilaplacian on lattices is studied. In [19], the eigenvalue estimates are used 
to the study the stability of discrete Schrödinger operators. For the continuous model, the eigenvalues and eigenfunctions of the discrete spectrum for Robin Laplacians are constructively computed in the paper [15]. In [6], the authors formulate a two-term asymptotic expansion of eigenvalue sums of the Laplacian on a bounded domain with Robin boundary conditions. They also prove the asymptotics in terms of semi-classical analysis. In this paper, we study properties of the eigenvalues of the discrete Laplacian operator with Robin boundary conditions; including the interlacing property, the first eigenvalue gap, and the monotonicity property. By deriving a secular equation of $(1.3)$, we first show in Theorem 3.6 that for $(\alpha, \beta)$ lying in a certain domain $\Omega_{1}$, the interlacing properties of eigenvalues holds:

$$
\lambda_{1}<\mu_{1}<\lambda_{2}<\mu_{2}<\cdots<\mu_{n-1}<\lambda_{n}
$$

Here, $\lambda_{j}$ is the $j$ th eigenvalue of $L(\alpha, \beta)$ and

$$
\mu_{j}=2-2 \cos \frac{j \pi}{n} \quad \text { for } j=1, \ldots, n-1
$$

For $(\alpha, \beta) \in \Omega_{1}$, we prove in Remark 4.7 the following first eigenvalue gap result:

$$
\lambda_{2}(\alpha, \beta)-\lambda_{1}(\alpha, \beta)>\frac{4}{n} \sin \left(\frac{\pi}{2 n}\right) \cos ^{-1}\left(\frac{1}{2} \sec \frac{\pi}{2 n}\right)=\frac{2 \pi^{2}}{3 n^{2}}+O\left(\frac{1}{n^{4}}\right) .
$$

Along some specific curves in the $(\alpha, \beta)$ space, the monotonicity property of eigenvalues is given in Theorem 5.1. Therefore, asymptotically as $n \gg 1$, the first eigenvalue gap of $n^{2} L(\alpha, \beta)$, which approximates the continuous operator $-d^{2} / d x^{2}$, is $2 \pi^{2} / 3$.

This paper is organized as follows. In Section 2 , we derive a secular equation of 1.3 . In Section 3 , we study the parameter space of $(\alpha, \beta)$ and use the space to study interlacing properties of eigenvalues. In Section 4 , we estimate the first eigenvalue gap. In Section 5 . monotonicity properties with respect to the parameter $(\alpha, \beta)$ are studied.

\section{The secular equation}

In this section, we derive the secular equation of $(1.3)$. Suppose that $\mathbf{u}=\left[u_{1}, u_{2}, \ldots, u_{n}\right]^{\top} \in$ $\mathbb{R}^{n}$ is an eigenvector of $L$ in 1.1 corresponding to eigenvalue $\lambda \in(0,4)$. Let $u_{k}=$ $c_{+} r^{k}+c_{-} r^{-k}$ for some nonzero complex conjugates $c_{+}, c_{-}$, and $k=1, \ldots, n$. By regarding $1.3 \mathrm{a}$ as a linear difference equation, we have the characteristic equation

$$
-1+2 r-r^{2}=\lambda r
$$

Letting $\delta=(2-\lambda) / 2$, the solutions of $(2.1)$ are $r_{ \pm}=\delta \pm \sqrt{\delta^{2}-1}$. Since $\lambda \in(0,4)$, we have $\delta \in(-1,1)$. Then there exists a $\theta \in(0, \pi)$ such that the roots of (2.1) are 
$r_{ \pm}=\cos \theta \pm i \sin \theta$. Since $\mathbf{u}$ is a real vector, and $c_{+}, c_{-} \in \mathbb{C}$ are conjugate, the $k$ th component of eigenvector $\mathbf{u}$ takes the form

$$
u_{k}=c_{+} e^{i k \theta}+c_{-} e^{-i k \theta}=c \cos k \theta+s \sin k \theta,
$$

where $c=2 \operatorname{Re}\left(c_{+}\right)$and $s=-2 \operatorname{Im}\left(c_{+}\right)$are real constants and are not all zero. Note that if $c$ and $s$ are not all zero, then $\mathbf{u} \neq \mathbf{0}$ because the vectors $[\cos \theta, \cos 2 \theta, \ldots, \cos k \theta]^{\top}$ and $[\sin \theta, \sin 2 \theta, \ldots, \sin k \theta]^{\top}$ are linearly independent.

Substituting equation 2.2 into $1.3 \mathrm{~b}$, we have the linear system

$$
\left[\begin{array}{cc}
\alpha \cos \theta-1 & \alpha \sin \theta \\
\beta \cos n \theta-\cos (n+1) \theta & \beta \sin n \theta-\sin (n+1) \theta
\end{array}\right]\left[\begin{array}{l}
c \\
s
\end{array}\right]=\mathbf{0}
$$

which has nontrivial solutions. Taking the determinant of the coefficient matrix and applying the compound-angle formula, we see that the angle $\theta$ must satisfy

$$
\begin{array}{r}
(\alpha \cos \theta-1)(\beta \sin n \theta-\sin n \theta \cos \theta-\cos n \theta \sin \theta) \\
-\alpha \sin \theta(\beta \cos n \theta-\cos n \theta \cos \theta+\sin n \theta \sin \theta)=0 .
\end{array}
$$

Then we have

$$
0=\sin n \theta[(1+\alpha \beta) \cos \theta-\alpha-\beta]+\cos n \theta[(1-\alpha \beta) \sin \theta] .
$$

Hence, the secular equation of $\sqrt{1.3}$ can be obtained as

$$
\cot n \theta=\frac{\alpha+\beta}{1-\alpha \beta} \frac{1}{\sin \theta}-\frac{1+\alpha \beta}{1-\alpha \beta} \cot \theta
$$

provided that $\alpha \beta \neq 1$ and $\sin \theta \neq 0$. The following theorem shows that the solution of the secular equation 2.5 can be used to determine the eigenvalue of $L$ in 1.3 .

Theorem 2.1. Given $\alpha, \beta \in \mathbb{R}$ with $\alpha \beta \neq 1$, let

$$
g(\theta)=A \csc \theta-B \cot \theta
$$

where

$$
A \equiv A(\alpha, \beta)=\frac{\alpha+\beta}{1-\alpha \beta}, \quad B \equiv B(\alpha, \beta)=\frac{1+\alpha \beta}{1-\alpha \beta} .
$$

Suppose that there is a $\theta_{*} \in(0, \pi)$ such that $g\left(\theta_{*}\right)=\cot n \theta_{*}$, i.e., the secular equation (2.5) holds at $\theta=\theta_{*}$, then the matrix $L(\alpha, \beta)$ in (1.1) has eigenvalue $\lambda=2-2 \cos \theta_{*}$.

Proof. Suppose that $\theta_{*} \in(0, \pi)$ is a solution of (2.5). Then the linear system (2.3) has a nontrivial solution $c_{*}$ and $s_{*}$. Since the real part of roots of 2.1 is $\cos \theta_{*}=(2-\lambda) / 2$, the value $\lambda=2-2 \cos \theta_{*}$ and the vector $\mathbf{u}=\left[u_{1}, u_{2}, \ldots, u_{n}\right]^{\top} \in \mathbb{R}^{n}$ can be constructed by 2.2 with $c=c_{*}$ and $s=s_{*}$. Note that $\mathbf{u}$ is a nonzero vector because $c_{*}$ and $s_{*}$ are not all zero. Then $\lambda$ and $\mathbf{u}$ satisfy $(1.3)$, and hence $\lambda$ is an eigenvalue of $L(\alpha, \beta)$ corresponding to eigenvector $\mathbf{u}$. 
The case of $\alpha \beta=1$, in which the Neumann condition is included, is studied in Theorem 2.2. In this case, we shall see that the eigenvalues and secular equation of $L(\alpha, \beta)$ are explicitly expressed.

Theorem 2.2. Suppose that $\alpha \beta=1$ and that $\theta_{*} \in(0, \pi)$ is a root of

$$
\sin n \theta=0,
$$

then the matrix $L=L(\alpha, \beta)$ in (1.1) has eigenvalue $\lambda=2-2 \cos \theta_{*}$. In addition, if $(\alpha, \beta)=(1,1)$, then $\lambda=0$ is an eigenvalue of $L$; and if $(\alpha, \beta)=(-1,-1)$, then $\lambda=4$ is an eigenvalue of $L$.

Proof. Suppose that $\alpha \beta=1$, then $(2.4)$ becomes $\sin n \theta[(1+\alpha \beta) \cos \theta-\alpha-\beta]=0$. This implies that $\sin n \theta=0$ or $2 \cos \theta=\alpha+\beta$. If $\theta_{*} \in(0, \pi)$ is a root of $\sin n \theta=0$, then $\lambda=2-2 \cos \theta_{*}$ is an eigenvalue of $L(\alpha, \beta)$. Since $\alpha \beta=1$, we see that $2 \cos \theta=\alpha+\beta$ holds when $(\alpha, \beta, \theta)=(1,1,0)$ and $(\alpha, \beta, \theta)=(-1,-1, \pi)$. In both cases, we take $c=1$ and $s=0$ in 2.2 . Then a nonzero eigenvector corresponding to eigenvalue $\lambda=0$ and $\lambda=4$ can be obtained for the case $\alpha=\beta=1$ and $\alpha=\beta=-1$, respectively.

Remark 2.3. (i) For the case $\alpha \beta \neq 1$, suppose that $0<\theta_{1}<\theta_{2}<\cdots<\theta_{\ell}<\pi$ are solutions of the secular equation $g(\theta)=\cot n \theta$. Let $\lambda_{j}=2-2 \cos \theta_{j}$ for $j=1, \ldots, \ell$. Theorem 2.1 shows that $0<\lambda_{1}<\lambda_{2}<\cdots<\lambda_{\ell}<4$ are eigenvalues of $L$.

(ii) For the case $\alpha \beta=1$, Theorem 2.2 shows that $L$ has $n-1$ eigenvalues $2-2 \cos (j \pi / n)$, for $j=1, \ldots, n-1$, inside the interval $(0,4)$. The rest eigenvalue of $L$ belongs to $(-\infty, 0]$ or $[4, \infty)$.

3. The parameter space and interlacing properties of eigenvalues

In Section 2, we see the solutions of secular equation $g(\theta)=\cot n \theta$ play an important role to determine the eigenvalues of $L$. In $(2.6)$, we see that the function $g(\theta)$ is dependent on the coefficients $A \equiv A(\alpha, \beta)$ and $B \equiv B(\alpha, \beta)$. By using the parameters $(A, B)$, computations in Section 3.2 can be simplified. In this section, we first investigate the parameter transformations and the interlacing property of eigenvalues.

\subsection{Partition of the parameter space and the parameter transformation}

It follows from (1.1) that the eigenvalues of matrices $L(\alpha, \beta)$ and $L(\beta, \alpha)$ are the same. Hence, to investigate the eigenvalues of $L$, we only consider the parameter $(\alpha, \beta) \in \mathbb{R}^{2}$ with $\alpha \leq \beta$. Let $\Omega=\Omega_{1} \cup \Omega_{2} \cup \Omega_{3}$, where

$$
\Omega_{1}=\left\{(\alpha, \beta) \in \mathbb{R}^{2} \mid \alpha \leq \beta \text { and } \alpha \beta<1\right\}
$$




$$
\begin{aligned}
& \Omega_{2}=\left\{(\alpha, \beta) \in \mathbb{R}^{2} \mid 0<\alpha \leq \beta \text { and } \alpha \beta>1\right\}, \\
& \Omega_{3}=\left\{(\alpha, \beta) \in \mathbb{R}^{2} \mid \alpha \leq \beta<0 \text { and } \alpha \beta>1\right\} .
\end{aligned}
$$

We consider the transformation $T: \Omega \rightarrow \mathbb{R}^{2}$ defined by

$$
T(\alpha, \beta) \equiv(A(\alpha, \beta), B(\alpha, \beta))=\left(\frac{\alpha+\beta}{1-\alpha \beta}, \frac{1+\alpha \beta}{1-\alpha \beta}\right) .
$$

In this subsection, we will characterize the images of $\Omega_{1}, \Omega_{2}$, and $\Omega_{3}$ under the transformation $T$. First, we show that the transformation is injective.

Lemma 3.1. The transformation $T: \Omega \rightarrow \mathbb{R}^{2}$ is injective.

Proof. Suppose that $T\left(\alpha_{1}, \beta_{1}\right)=T\left(\alpha_{2}, \beta_{2}\right)$. Using $B\left(\alpha_{1}, \beta_{1}\right)=B\left(\alpha_{2}, \beta_{2}\right)$ in (3.2), we have $\left(1+\alpha_{1} \beta_{1}\right)\left(1-\alpha_{2} \beta_{2}\right)=\left(1+\alpha_{2} \beta_{2}\right)\left(1-\alpha_{1} \beta_{1}\right)$. This leads to $\alpha_{1} \beta_{1}=\alpha_{2} \beta_{2}$. Applying $A\left(\alpha_{1}, \beta_{1}\right)=A\left(\alpha_{2}, \beta_{2}\right)$ and $\alpha_{1} \beta_{1}=\alpha_{2} \beta_{2}$, we obtain $\alpha_{1}+\beta_{1}=\alpha_{2}+\beta_{2}$. Together with the facts that $\alpha_{1} \beta_{1}=\alpha_{2} \beta_{2}, \alpha_{1} \leq \beta_{1}$ and $\alpha_{2} \leq \beta_{2}$, we have $\alpha_{1}=\alpha_{2}$ and $\beta_{1}=\beta_{2}$. Hence, the transformation $T$ is injective.

The following lemmas describe the sets of $T\left(\Omega_{1}\right), T\left(\Omega_{2}\right)$, and $T\left(\Omega_{3}\right)$.

Lemma 3.2. Let $h(x)=\sqrt{1+x^{2}}$. Then the set $T\left(\Omega_{1}\right)$ is

$$
T\left(\Omega_{1}\right)=\left\{(A, B) \in \mathbb{R}^{2} \mid-1<B \leq h(A)\right\} .
$$

Proof. Let $\mathcal{C}=\{(\alpha, \alpha) \mid-1<\alpha<1\}$. Then $\mathcal{C}$ is some part of the boundary of $\Omega_{1}$. Let $\mathbf{0}=(0,0) \in \mathcal{C}$ be the origin. Then $T(\mathbf{0})=(0,1)$ and the image of $\mathcal{C}$ under $T$ is

$$
T(\mathcal{C})=\left\{\left(\frac{2 \alpha}{1-\alpha^{2}}, \frac{1+\alpha^{2}}{1-\alpha^{2}}\right) \mid-1<\alpha<1\right\} .
$$

Since $-1<\alpha<1, A=2 \alpha /\left(1-\alpha^{2}\right)$ can be arbitrary real number and

$$
h(A)=\sqrt{1+A^{2}}=\frac{1+\alpha^{2}}{1-\alpha^{2}} .
$$

Hence, $T(\mathcal{C})=\left\{(A, B) \in \mathbb{R}^{2} \mid B=h(A)\right\}$.

From (3.1), we have $\Omega_{1}=\Omega_{1,1} \cup \Omega_{1,2} \cup \Omega_{1,3}$, where

$$
\begin{aligned}
& \Omega_{1,1}=\{(\alpha, k \alpha) \mid 1 \leq k \text { and } 0<\alpha<\sqrt{1 / k}\} \\
& \Omega_{1,2}=\{(\alpha, \beta) \mid \alpha \leq 0 \text { and } 0 \leq \beta\} \\
& \Omega_{1,3}=\{(\alpha, k \alpha) \mid 0<k \leq 1 \text { and }-\sqrt{1 / k}<\alpha<0\} .
\end{aligned}
$$

See Figure 3.1 for the illustration. Let $\mathcal{S}_{+}=\{(0, \beta) \mid \beta \geq 0\}$ and $\mathcal{S}_{-}=\{(\alpha, 0) \mid \alpha \leq 0\}$. Then $\mathcal{S}_{+}$is the intersection of the boundaries of $\Omega_{1,1}$ and $\Omega_{1,2}$, and $\mathcal{S}_{-}$is the intersection of the boundaries of $\Omega_{1,2}$ and $\Omega_{1,3}$. It is easy to see that

$$
T\left(\mathcal{S}_{+}\right)=\{(A, 1) \mid A \geq 0\} \quad \text { and } \quad T\left(\mathcal{S}_{-}\right)=\{(A, 1) \mid A \leq 0\} .
$$


In the following, we will describe the images of $\Omega_{1, i}$ for $i=1,2,3$ under $T$. The images have been illustrated in Figure 3.1 .
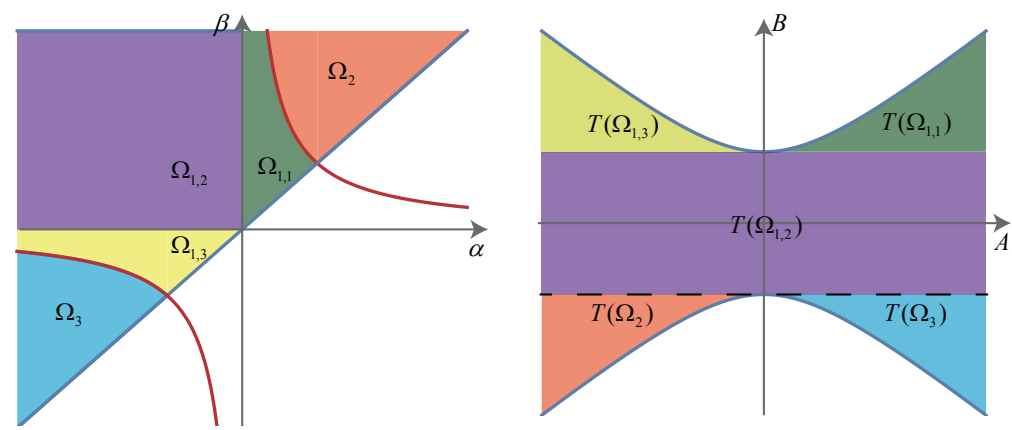

Figure 3.1: The domains $\Omega_{1}, \Omega_{2}$ and $\Omega_{3}$ and the images under $T$.

(i) For each $k \geq 1$, the curve $\mathcal{S}_{k}=\{(\alpha, k \alpha) \mid 0<\alpha<\sqrt{1 / k}\} \subseteq \Omega_{1,1}$. Then $T\left(\mathcal{S}_{k}\right)=$ $\left\{\left(\frac{(k+1) \alpha}{1-k \alpha^{2}}, \frac{1+k \alpha^{2}}{1-k \alpha^{2}}\right) \mid 0<\alpha<\sqrt{1 / k}\right\}$ is a curve from $T(\mathbf{0})=(0,1)$ to $\lim _{\alpha \rightarrow \sqrt{1 / k}}-\left(\frac{(k+1) \alpha}{1-k \alpha^{2}}\right.$, $\left.\frac{1+k \alpha^{2}}{1-k \alpha^{2}}\right)=(\infty, \infty)$ and

$$
h\left(\frac{(k+1) \alpha}{1-k \alpha^{2}}\right)=\frac{\sqrt{\left(1-k \alpha^{2}\right)^{2}+((k+1) \alpha)^{2}}}{1-k \alpha^{2}} \geq \frac{1+k \alpha^{2}}{1-k \alpha^{2}}
$$

because $k^{2}+1 \geq 2 k$. It follows from (3.4), 3.5), and Lemma 3.1 that

$$
T\left(\Omega_{1,1}\right)=\{(A, B) \mid 0<A, 1<B \leq h(A)\} .
$$

(ii) We show that

$$
T\left(\Omega_{1,2}\right)=\{(A, B) \mid A \in \mathbb{R},-1<B \leq 1\} .
$$

For each $(A, B) \in T\left(\Omega_{1,2}\right)$, there exists $(\alpha, \beta) \in \Omega_{1,2}$ such that $A=(\alpha+\beta) /(1-\alpha \beta)$ and $B=(1+\alpha \beta) /(1-\alpha \beta)$. Since $(\alpha, \beta) \in \Omega_{1,2}$ and $\alpha \beta \leq 0$, this leads that $-1<B \leq 1$. This proves the inclusion.

For each pair $(A, B)$ with $A \in \mathbb{R}$ and $-1<B \leq 1$, there exists a number $x$ with $x \leq 0$ such that equation $(1+x) /(1-x)=B$ holds. Consider the system of equations

$$
\alpha \beta=x, \quad \alpha+\beta=(1-x) A .
$$

Since $\alpha \beta=x$ is hyperbola in second quadrant and $\alpha+\beta=(1-x) A$ is a line with slope -1 , the system has unique solution $(\alpha, \beta)$ with $\alpha \leq 0$ and $\beta \geq 0$. This proves the conclusion.

(iii) For each $0<k \leq 1$, the curve $\mathcal{S}_{k}=\{(\alpha, k \alpha) \mid-\sqrt{1 / k}<\alpha<0\} \subseteq \Omega_{1,3}$. Then $T\left(\mathcal{S}_{k}\right)=\left\{\left(\frac{(k+1) \alpha}{1-k \alpha^{2}}, \frac{1+k \alpha^{2}}{1-k \alpha^{2}}\right) \mid-\sqrt{1 / k}<\alpha<0\right\}$ is a curve from $T(\mathbf{0})=(0,1)$ to 
$\lim _{\alpha \rightarrow-\sqrt{1 / k}^{+}}\left(\frac{(k+1) \alpha}{1-k \alpha^{2}}, \frac{1+k \alpha^{2}}{1-k \alpha^{2}}\right)=(-\infty, \infty)$. It follows from (3.4), 3.5), and Lemma 3.1 that

$$
T\left(\Omega_{1,3}\right)=\{(A, B) \mid A<0,1<B \leq h(A)\} .
$$

Combining (3.6), (3.7), and (3.8), we obtain (3.3).

Lemma 3.3. Let $h(x)=\sqrt{1+x^{2}}$. Then the sets $T\left(\Omega_{2}\right)$ and $T\left(\Omega_{3}\right)$ are

$$
\begin{aligned}
& T\left(\Omega_{2}\right)=\left\{(A, B) \in \mathbb{R}^{2} \mid A<0,-h(A) \leq B<-1\right\}, \\
& T\left(\Omega_{3}\right)=\left\{(A, B) \in \mathbb{R}^{2} \mid A>0,-h(A) \leq B<-1\right\} .
\end{aligned}
$$

Proof. First, we show that

$$
T\left(\Omega_{2}\right)=\left\{(A, B) \in \mathbb{R}^{2} \mid A<0,-h(A) \leq B<-1\right\} .
$$

For each $(A, B) \in T\left(\Omega_{2}\right)$, there exist $\alpha$ and $\beta$ with $0<\alpha \leq \beta$ and $\alpha \beta>1$ such that $A=(\alpha+\beta) /(1-\alpha \beta)$ and $B=(1+\alpha \beta) /(1-\alpha \beta)$. It is easily seen that $A<0$ and $B<-1$. Furthermore,

$$
h(A)=\frac{\sqrt{(1-\alpha \beta)^{2}+(\alpha+\beta)^{2}}}{\alpha \beta-1} \geq \frac{1+\alpha \beta}{\alpha \beta-1}=-B .
$$

Hence, $A<0$ and $-h(A) \leq B<-1$. This proves the inclusion.

For each pair $(A, B)$ with $A<0$ and $-h(A) \leq B<-1, x=(B-1)(1+B)>1$ is the unique solution of $(1+x) /(1-x)=B$. To prove $(A, B) \in T\left(\Omega_{2}\right)$, it suffices to show the system of equations

$$
\alpha \beta=x, \quad \alpha+\beta=(1-x) A
$$

has solution $(\alpha, \beta) \in \Omega_{2}$. We see that $\alpha \beta=x$ is hyperbola in first quadrant and $\alpha+\beta=$ $(1-x) A$ is a line with slope -1 , hence, the system 3.10 having solution $(\alpha, \beta)$ with $\alpha \leq \beta$ is equivalent to $(1-x) A \geq 2 \sqrt{x}$.

Since $-h(A) \leq B$, we have $1+A^{2} \geq B^{2}$. This implies that $A^{2} \geq(B+1)(B-1)=$ $x(B+1)^{2}$. Since $(1-x)^{2}=\left(\frac{2}{1+B}\right)^{2}$, we have $(1-x)^{2} A^{2} \geq 4 x$. Hence, 3.10 has solution with $\alpha \leq \beta$. This proves the conclusion.

Similarly, we can show that $T\left(\Omega_{3}\right)=\left\{(A, B) \in \mathbb{R}^{2} \mid A>0,-h(A) \leq B<-1\right\}$. Hence, (3.9) holds.

We have characterized the image of the parameter domain $\Omega$ in (3.1) under the oneto-one parameter transformation $T$. The relation between the domain and image have been illustrated in Figure 3.1. In the next subsection, we shall investigate the interlacing properties of eigenvalues of $L$ by the secular equation with parameter $(A, B) \in T(\Omega)$. 


\subsection{Interlacing properties of eigenvalues}

Theorem 2.1 shows that if $\theta_{*} \in(0, \pi)$ is a solution of secular equation $g(\theta)=\cot n \theta$, then $L$ in (1.1) has eigenvalue $\lambda=2-2 \cos \theta_{*}$. In this subsection, we will investigate interlacing properties of eigenvalues and the numbers of eigenvalues in $[0,4]$ for each parameter $(A, B) \in T(\Omega)$.

Let $(A, B) \in T(\Omega)$ with $B \neq 0$. Denote $K=A / B$. If $K \in(-1,1)$, then there exists a $\theta_{K} \in(0, \pi)$ such that $\cos \theta_{K}=K$. Then we have $g\left(\theta_{K}\right)=0$. By using

$$
g^{\prime}(\theta)=B\left(\frac{1-K \cos \theta}{\sin ^{2} \theta}\right)=\frac{B-A \cos \theta}{\sin ^{2} \theta},
$$

we have the following consequence immediately.

Lemma 3.4. The function $g(\theta)$ defined in $(2.6)$ is continuous on $(0, \pi)$. When $B=0$, $g(\theta)=A \csc \theta$. If $B \neq 0$ and set $K=A / B$, then the following holds true.

(a) For the case $-1<K<1$ :

(i) If $B>0$, then $g(\theta)$ is increasing and $\lim _{\theta \rightarrow 0^{+}} g(\theta)=-\infty, \lim _{\theta \rightarrow \pi^{-}} g(\theta)=\infty$;

(ii) If $B<0$, then $g(\theta)$ is decreasing and $\lim _{\theta \rightarrow 0^{+}} g(\theta)=\infty, \lim _{\theta \rightarrow \pi^{-}} g(\theta)=-\infty$.

(b) For the case $K<-1$ or $K>1$ : there is a $\theta_{1 / K} \in(0, \pi)$ such that $\cos \theta_{1 / K}=1 / K$.

(i) If $A>0$, then $g(\theta)$ is decreasing on $\left(0, \theta_{1 / K}\right]$ and increasing on $\left[\theta_{1 / K}, \pi\right)$. In addition, $\lim _{\theta \rightarrow 0^{+}} g(\theta)=\infty$ and $\lim _{\theta \rightarrow \pi^{-}} g(\theta)=\infty$;

(ii) If $A<0$, then $g(\theta)$ is increasing on $\left(0, \theta_{1 / K}\right]$ and decreasing on $\left[\theta_{1 / K}, \pi\right)$. In addition, $\lim _{\theta \rightarrow 0^{+}} g(\theta)=-\infty$ and $\lim _{\theta \rightarrow \pi^{-}} g(\theta)=-\infty$.

(c) For the case $K= \pm 1$ :

(i) If $K=1$, then $\lim _{\theta \rightarrow 0^{+}} g(\theta)=0$. In addition, if $B>0$, then $g(\theta)$ is increasing and $\lim _{\theta \rightarrow \pi^{-}} g(\theta)=\infty$; if $B<0$, then $g(\theta)$ is decreasing and $\lim _{\theta \rightarrow \pi^{-}} g(\theta)=$ $-\infty$;

(ii) If $K=-1$, then $\lim _{\theta \rightarrow \pi^{-}} g(\theta)=0$. In addition, if $B>0$, then $g(\theta)$ is increasing and $\lim _{\theta \rightarrow 0^{+}} g(\theta)=-\infty$; if $B<0$, then $g(\theta)$ is decreasing and $\lim _{\theta \rightarrow 0^{+}} g(\theta)=\infty$.

The secular equation can only be applied to determine the eigenvalues inside the open interval $(0,4)$. Next, we determine whether $L$ has eigenvalue $\lambda=0$ or $\lambda=4$.

Lemma 3.5. Let $(A, B)=T(\alpha, \beta) \in T(\Omega)$. The matrix $L$ in (1.1) has $\lambda=0$ eigenvalue if and only if $A-B=1 / n$. L has $\lambda=4$ eigenvalue if and only if $A+B=-1 / n$. 
Proof. Suppose that $\lambda=0$ is an eigenvalue of $L=L(\alpha, \beta)$ in $(1.1)$. Then the characteristic equation (2.1) has solution $r=1$ with multiplicity 2 . Then the $k$ th component of eigenvector $\mathbf{u}$ has the form $u_{k}=c+d k$, where $c, d \in \mathbb{R}$ are not all zero. Substituting this formula into $1.3 \mathrm{~b}$, we have

$$
\left[\begin{array}{cc}
\alpha-1 & \alpha \\
\beta-1 & \beta n-n-1
\end{array}\right]\left[\begin{array}{l}
c \\
d
\end{array}\right]=\mathbf{0} .
$$

There are nonzero numbers $c$ and $d$ such that above equation holds if and only if the determinant of the coefficient matrix is zero, i.e., $\alpha \beta n-(n+1) \alpha-\beta n+n+1-\alpha \beta+\alpha=0$. This leads to $A-B=1 / n$.

The proof of rest argument is similar to the above procedure of proof.

Since the function $g(\theta)$ in $(2.6)$ is continuous on $(0, \pi)$, it follows from Lemma 3.4 that the secular equation $g(\theta)=\cot n \theta$ has at least $n-2$ distinct roots in $\left(\frac{\pi}{n}, \frac{(n-1) \pi}{n}\right)$. In addition, by using Theorem 2.1, we see that there are at least $n-2$ distinct eigenvalues in the interval $\left(\mu_{1}, \mu_{n-1}\right)$, where $\mu_{1}$ and $\mu_{n-1}$ are given in (1.4). In the following, we shall study interlacing properties for $\lambda_{j}$.

- For the case $(A, B) \in T\left(\Omega_{1}\right)=\left\{(A, B) \in \mathbb{R}^{2} \mid-1<B \leq h(A)\right\}$ :

Theorem 3.6. For each $(A, B) \in T\left(\Omega_{1}\right)$, the following interlacing property holds

$$
\lambda_{1}<\mu_{1}<\lambda_{2}<\mu_{2}<\cdots<\mu_{n-1}<\lambda_{n},
$$

where $\mu_{j}$ is given in (1.4). In addition,

(i) if $A-B<1 / n$, then $\lambda_{1}>0$; and if $A-B>1 / n$ then $\lambda_{1}<0$;

(ii) if $A+B<-1 / n$, then $\lambda_{n}>4$; and if $A+B>-1 / n$ then $\lambda_{n}<4$.

Proof. From Lemma 3.4, we see that $g(\theta)$ is continuous on $(0, \pi)$, hence, there are at least $n-2$ distinct roots $\theta_{j} \in\left(\frac{(j-1) \pi}{n}, \frac{j \pi}{n}\right)$, for $j=2, \ldots, n-1$, of $g(\theta)=\cot n \theta$. From Theorem 2.1. $\lambda_{j}=2-2 \cos \theta_{j}$ are eigenvalues of $L$ with $\mu_{1}<\lambda_{2}<\mu_{2}<\cdots<\lambda_{n-1}<\mu_{n-1}$. Now, we consider the solutions of $g(\theta)=\cot n \theta$ on $(0, \pi / n)$ and on $\left(\frac{(n-1) \pi}{n}, \pi\right)$.

Since $g(\theta)$ and $\cot n \theta$ are continuous on $(0, \pi / n)$ and

$$
\lim _{\theta \rightarrow 0^{+}} \cot n \theta=\infty, \quad \lim _{\theta \rightarrow \frac{\pi}{n}^{-}} \cot n \theta=-\infty
$$

$g(\theta)=\cot n \theta$ has a solution on $(0, \pi / n)$ if $\lim _{\theta \rightarrow 0^{+}} g(\theta) \neq \infty$. From Lemma 3.4 , we see that if $A=0$ and $B=1$ then $\lim _{\theta \rightarrow 0^{+}} g(\theta)=-\infty$, and hence, the secular equation has a solution on $(0, \pi / n)$. Using the continuity of eigenvalues and Lemma 3.5, we obtain that 
if $A-B<1 / n$, then the eigenvalue $\lambda_{1}>0$. On the other hand, Let $\alpha_{*}=-4$ and $\beta_{*}=4$. Then $A=\left(\alpha_{*}+\beta_{*}\right) /\left(1-\alpha_{*} \beta_{*}\right)=0$ and $B=\left(1+\alpha_{*} \beta_{*}\right) /\left(1-\alpha_{*} \beta_{*}\right)=-15 / 17$. Using Gershgorin disk theorem on $L=L\left(\alpha_{*}, \beta_{*}\right)$, the matrix $L$ has an eigenvalue $\lambda_{1}<0$ and an eigenvalue $\lambda_{n}>4$. Using the continuity of eigenvalues and Lemma 3.5 again, we obtain that if $A-B>1 / n$, then the eigenvalue $\lambda_{1}<0$.

Similarly, if $\lim _{\theta \rightarrow \pi^{-}} g(\theta) \neq-\infty$ then $g(\theta)=\cot n \theta$ has a solution on $\left(\frac{(n-1) \pi}{n}, \pi\right)$. From Lemma 3.4. we see that the $g(\theta)=\cot n \theta$ has a solution on $\left(\frac{(n-1) \pi}{n}, \pi\right)$ when $A=0$ and $B=1$. Hence, if $A+B>-1 / n$, then the eigenvalue $\lambda_{n}<4$. When $A=0$ and $B=-15 / 17$, we see that $L$ has an eigenvalue $\lambda_{n}>4$. Thus, if $A+B<-1 / n$, then the eigenvalue $\lambda_{n}>4$ by the continuity of eigenvalue. This completes the proof.

- For the case $(A, B) \in T\left(\Omega_{2}\right)=\left\{(A, B) \in \mathbb{R}^{2} \mid A<0,-h(A) \leq B<-1\right\}$ :

Lemma 3.7. If $(A, B) \in T\left(\Omega_{2}\right)$, then $\lambda_{1}<0$ and $\lambda_{n}<\mu_{n-1}$.

Proof. First, we show that $\lambda_{n}<\mu_{n-1}$. Suppose that $(A, B) \in T\left(\Omega_{2}\right)$. There exists $(\alpha, \beta) \in \Omega_{2}$ such that $A=(\alpha+\beta) /(1-\alpha \beta)$ and $B=(1+\alpha \beta) /(1-\alpha \beta)$. Since $(\alpha, \beta) \in$ $\Omega_{2}=\left\{(\alpha, \beta) \in \mathbb{R}^{2} \mid 0<\alpha \leq \beta\right.$ and $\left.\alpha \beta>1\right\}$, all eigenvalues of the matrix $L=L(\alpha, \beta)$ in (1.1) are smaller than 4 by Gershgorin disk theorem. In addition, 4 is not a eigenvalue of $L$. Hence, we have $\lambda_{n}<4$. From (3.9), we see that $A<0$ and $B<-1$. Using the fact that $A \csc \theta<0$ and $\cot \theta<\cot n \theta$ for $\theta \in\left(\frac{n-1}{n} \pi, \pi\right)$, we have

$$
g(\theta)=A \csc \theta-B \cot \theta<\cot n \theta \quad \text { for } \theta \in\left(\frac{n-1}{n} \pi, \pi\right) .
$$

That is, the secular equation $g(\theta)=\cot n \theta$ has no solution on $\left(\frac{n-1}{n} \pi, \pi\right)$. Hence, we have $\lambda_{n}<\mu_{n-1}$.

Next, we show that $\lambda_{1}<0$. Suppose that $\left(\alpha_{*}, \beta_{*}\right) \in \Omega_{2}=\left\{(\alpha, \beta) \in \mathbb{R}^{2} \mid 0<\alpha \leq\right.$ $\beta$ and $\alpha \beta>1\}$. Let

$$
\widehat{\alpha}=\min \left\{\alpha_{*}, 1\right\} \quad \text { and } \quad \widehat{\beta}=2-\widehat{\alpha} \text {. }
$$

We have $\widehat{\alpha} \leq 1 \leq \widehat{\beta}$ and $\widehat{\alpha}+\widehat{\beta}=2$. Here,

$$
(\widehat{\alpha}, \widehat{\beta})= \begin{cases}\left(\alpha_{*}, 2-\alpha_{*}\right), & \alpha_{*} \leq 1 \\ (1,1), & \alpha_{*}>1\end{cases}
$$

Since $\alpha+\beta=2$ is the tangent line to the curve $\{(\alpha, \beta) \mid \alpha \beta=1\}$ at the point $(1,1)$ and $\left(\alpha_{*}, \beta_{*}\right) \in \Omega_{2}$, this implies $2-\alpha_{*}<\beta_{*}$ and $1<\beta_{*}$. From (3.12), we have $\widehat{\beta}<\beta_{*}$. In addition, $\widehat{\alpha} \widehat{\beta} \leq 1$. Hence,

$$
\widehat{\alpha} \leq \alpha_{*}, \quad 1 \leq \widehat{\beta}<\beta_{*}, \quad \text { and } \quad \widehat{\alpha} \widehat{\beta} \leq 1 .
$$


Let $\widehat{L}=L(\widehat{\alpha}, \widehat{\beta}) \in \mathbb{R}^{n \times n}$ be defined in 1.1$)$ and $\widehat{\lambda}_{1}$ be the smallest eigenvalue of $\widehat{L}$. We have $\lambda_{1}<\widehat{\lambda}_{1}$ (see [17, Theorem 2.7]). To show $\lambda_{1}<0$, it suffices to show that $\widehat{\lambda}_{1} \leq 0$.

(i) Suppose that $\widehat{\alpha} \neq 1$. From (3.11), we have $\widehat{\alpha} \widehat{\beta}<1$. Then $(\widehat{\alpha}, \widehat{\beta}) \in \Omega_{1}$ in (3.1). Using (3.11) again, we have $\widehat{A}=(\widehat{\alpha}+\widehat{\beta}) /(1-\widehat{\alpha} \widehat{\beta})=2 /(1-\widehat{\alpha})^{2}$ and $\widehat{B}=(1+\widehat{\alpha} \widehat{\beta}) /(1-\widehat{\alpha} \widehat{\beta})=$ $\left(1+2 \widehat{\alpha}-\widehat{\alpha}^{2}\right) /(1-\widehat{\alpha})^{2}$. Then we obtain that $\widehat{A}-\widehat{B}=1>1 / n$. It follows from Theorem 3.6 that $\widehat{\lambda}_{1}<0$.

(ii) Suppose that $\widehat{\alpha}=1$. Then $\widehat{\beta}=1$ by 3.11 . We see that $\widehat{\lambda}_{1}=0$ by Theorem 2.2. This completes the proof.

Theorem 3.8. For each $(A, B) \in T\left(\Omega_{2}\right)$, we have $\lambda_{1}<0$ and the interlacing property

$$
\lambda_{2}<\mu_{1}<\lambda_{3}<\mu_{2}<\cdots<\mu_{n-2}<\lambda_{n}<\mu_{n-1} .
$$

In addition, if $A-B<1 / n$, then $\lambda_{2}>0$; and if $A-B>1 / n$, then $\lambda_{2}<0$.

Proof. The fact $\lambda_{1}<0$ follows from Lemma 3.7 directly. The interlacing property $\lambda_{2}<$ $\mu_{1}<\lambda_{3}<\mu_{2}<\cdots<\mu_{n-2}<\lambda_{n}<\mu_{n-1}$ is a consequence of Theorem 2.1. Suppose that $(A, B) \in T\left(\Omega_{2}\right)$ with $A<B<-1$. Then $K=A / B>1$. From Lemma 3.4, we have $\lim _{\theta \rightarrow 0^{-}} g(\theta)=-\infty$. In this case, the secular equation $g(\theta)=\cot n \theta$ has a $\operatorname{root}$ on $(0, \pi / n)$, hence, $\lambda_{2}>0$ by Theorem 2.1. Using the continuity of eigenvalues and Lemma 3.5, we obtain that if $A-B<1 / n$, then the eigenvalue $\lambda_{2}>0$.

Let $\alpha_{*}=\beta_{*}=4$, then $\left(\alpha_{*}, \beta_{*}\right) \in \Omega_{2}$ and the matrix $L$ has two negative eigenvalues, $\lambda_{1}$ and $\lambda_{2}$, by the Gershgorin disk theorem. In this case, $A=\left(\alpha_{*}+\beta_{*}\right) /\left(1-\alpha_{*} \beta_{*}\right)=-8 / 15$, $B=\left(1+\alpha_{*} \beta_{*}\right) /\left(1-\alpha_{*} \beta_{*}\right)=-17 / 15$ and hence $A-B=9 / 15>1 / n$. Using the continuity of eigenvalues and Lemma 3.5, we obtain that if $A-B>1 / n$, then the eigenvalue $\lambda_{2}<0$.

- For the case $(A, B) \in T\left(\Omega_{3}\right)=\left\{(A, B) \in \mathbb{R}^{2} \mid A>0,-h(A) \leq B<-1\right\}$ :

A similar proof as that of Theorem 3.8 and applying Theorem 2.2 give the following assertion.

Theorem 3.9. For each $(A, B) \in T\left(\Omega_{3}\right)$, we have $4<\lambda_{n}$ and the interlacing property

$$
\mu_{1}<\lambda_{1}<\mu_{2}<\lambda_{2}<\cdots<\mu_{n-1}<\lambda_{n-1} .
$$

In addition, if $A+B>-1 / n$, then $\lambda_{n-1}<4$; and if $A+B<-1 / n$, then $4<\lambda_{n-1}$.

Remark 3.10. We give some comments on the interlacing property in this section. The interlacing theorem in [7, p. 183] states that Let $A \in \mathbb{R}^{n \times n}$ be symmetric, $\mathbf{z} \in \mathbb{R}^{n}$ and let the eigenvalues $\lambda_{i}(A)$ and $\lambda_{i}\left(A \pm \mathbf{z z}^{\top}\right)$ be arranged in increasing order. Then

$$
\begin{gathered}
\lambda_{1}(A) \leq \lambda_{1}\left(A+\mathbf{z z}^{\top}\right) \leq \lambda_{2}(A) \leq \cdots \leq \lambda_{n}(A) \leq \lambda_{n}\left(A+\mathbf{z z}^{\top}\right), \\
\lambda_{1}\left(A-\mathbf{z z}^{\top}\right) \leq \lambda_{1}(A) \leq \lambda_{2}\left(A-\mathbf{z z}^{\top}\right) \leq \cdots \leq \lambda_{n}\left(A-\mathbf{z z}^{\top}\right) \leq \lambda_{n}(A) .
\end{gathered}
$$


Some interlacing properties of the eigenvalues in Section 3.2 can be obtained by employing the interlacing theorem. From Remark 2.3(ii), the Laplacian matrix $L(\alpha, \beta)$ with $\alpha \beta=1$ (the red curve in the left figure of Figure 3.1 has eigenvalues $\mu_{j}=2-2 \cos \frac{j \pi}{n}$ for $j=$ $1, \ldots, n-1$. Application of the above interlacing theorem can lead to some interlacing properties of eigenvalues. Let $\mathbf{e}_{1}$ and $\mathbf{e}_{n}$ denote the first and the last columns of the identity matrix, respectively.

- For $(\alpha, \beta) \in\left\{(\alpha, \beta) \in \mathbb{R}^{2} \mid \alpha \leq \beta\right.$ and $\left.\beta \geq 1\right\}$, we set $A=L(1 / \beta, \beta)$ and $\mathbf{z}=\mathbf{e}_{1}$ in the interlacing theorem.

- For $(\alpha, \beta) \in\left\{(\alpha, \beta) \in \mathbb{R}^{2} \mid \alpha \leq \beta\right.$ and $\left.\alpha \leq-1\right\}$, we set $A=L(\alpha, 1 / \alpha)$ and $\mathbf{z}=\mathbf{e}_{n}$ in the interlacing theorem.

If $(\alpha, \beta)$ belongs to the region $\left\{(\alpha, \beta) \in \mathbb{R}^{2} \mid-1 \leq \alpha \leq 0\right.$ and $\left.0 \leq \beta \leq 1\right\} \subseteq \Omega_{1}$, then it seems not easy to obtain the interlacing properties in Theorem 3.6 by using the interlacing theorem.

The secular equation is constructed by using the structure and the entries of Laplacian matrix $L(\alpha, \beta)$. It can be used to estimate accurately the location of eigenvalues of $L(\alpha, \beta)$. Using the secular equation, we show the interlacing properties of eigenvalues of $L(\alpha, \beta)$ for ( $\alpha, \beta)$ belonging $\Omega_{1} \cup \Omega_{2} \cup \Omega_{3}$. Furthermore, we can use the secular equation to estimate the first and second eigenvalues, $\lambda_{1}(\alpha, \beta)$ and $\lambda_{2}(\alpha, \beta)$, of the Laplacian matrix $L(\alpha, \beta)$. In the next section, we shall give a lower bound of $\lambda_{2}(\alpha, \beta)-\lambda_{1}(\alpha, \beta)$ for $(\alpha, \beta) \in \Omega_{1}$.

\section{Eigenvalue gaps}

In this section, we will estimate the gap of the first two eigenvalues of $L(\alpha, \beta)$ in 1.1 for the parameter $(\alpha, \beta) \in \Omega_{1}$, where $\Omega_{1}=\left\{(\alpha, \beta) \in \mathbb{R}^{2} \mid \alpha \leq \beta\right.$ and $\left.\alpha \beta<1\right\}$ is defined in (3.1). Namely, we will study a lower bound of

$$
\min _{(\alpha, \beta) \in \Omega_{1}} \lambda_{2}(\alpha, \beta)-\lambda_{1}(\alpha, \beta)
$$

To this end, we partition $\Omega_{1}$ into 3 parts:

$$
\begin{aligned}
& \Phi_{1}=\left\{(\alpha, \beta) \in \mathbb{R}^{2} \mid-1 \leq \alpha \leq \beta \leq 1 \text { and } \alpha \beta<1\right\} \\
& \Phi_{2}=\left\{(\alpha, \beta) \in \mathbb{R}^{2} \mid \alpha<-1, \beta \leq 1 \text { and } \alpha \beta<1\right\} \\
& \Phi_{3}=\left\{(\alpha, \beta) \in \mathbb{R}^{2} \mid \beta>1 \text { and } \alpha \beta<1\right\} .
\end{aligned}
$$

Here, the the three regions $\Phi_{i}, i=1,2,3$ are plotted in the left of Figure 4.1. Let $\mathcal{S}_{1}=\{(-1, \beta) \mid \beta>-1\}$ and $\mathcal{S}_{2}=\{(\alpha, 1) \mid \alpha<1\}$ be lines in $\Omega_{1}$. The images of these two lines under the transformation $T$ in $(3.2)$ are $T\left(\mathcal{S}_{1}\right)=\{(A,-A) \mid A<1\}$ and 
$T\left(\mathcal{S}_{2}\right)=\{(A, A) \mid A>-1\}$. From Lemma 3.2, we have

$$
\begin{aligned}
& T\left(\Phi_{1}\right)=\left\{(A, B) \in \mathbb{R}^{2}|| A \mid \leq B \leq h(A)\right\}, \\
& T\left(\Phi_{2}\right)=\left\{(A, B) \in \mathbb{R}^{2} \mid-1<B<-A \text { and } A \leq B\right\} \\
& T\left(\Phi_{3}\right)=\left\{(A, B) \in \mathbb{R}^{2} \mid-1<B<A\right\}
\end{aligned}
$$

where $h(x)=\sqrt{1+x^{2}}$ (see Figure 4.1 for the illustration). In the following, we shall investigate the lower bound of $\lambda_{2}-\lambda_{1}$ for $(\alpha, \beta)$ belonging $\Phi_{1}, \Phi_{2}$, and $\Phi_{3}$.
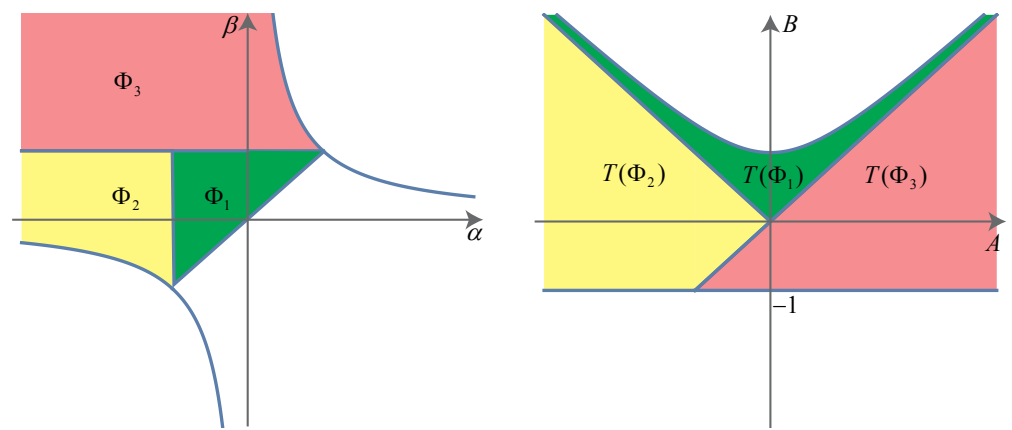

Figure 4.1: The domains $\Phi_{1}, \Phi_{2}$ and $\Phi_{3}$ and the images under $T$.

- For the case $(\alpha, \beta) \in \Phi_{1}=\left\{(\alpha, \beta) \in \mathbb{R}^{2} \mid-1 \leq \alpha \leq \beta \leq 1\right.$ and $\left.\alpha \beta<1\right\}$ :

Suppose that $(\alpha, \beta) \in \Phi_{1}$. From 4.2 and Theorem 3.6, we see that

$$
0<\lambda_{1}=2-2 \cos \theta_{1}<\mu_{1}<\lambda_{2}=2-2 \cos \theta_{2}<\mu_{2}
$$

where $\theta_{1}<\theta_{2}$ are the two smallest roots of secular equation $g(\theta)=\cot n \theta$. The region $T\left(\Phi_{1}\right)$ in 4.2 can be rewritten as

$$
T\left(\Phi_{1}\right)=\left\{(K B, B) \mid-1 \leq K \leq 1 \text { and } 0 \leq B \leq \sqrt{1+K^{2} B^{2}}\right\} .
$$

In other words, for $(A, B)=T(\alpha, \beta) \in T\left(\Phi_{1}\right)$, there is a $K_{*}$ with $-1 \leq K_{*} \leq 1$ such that $A=K_{*} B$ and $0 \leq B \leq \sqrt{1+K_{*}^{2} B^{2}}$. Now, let $K_{*}$ be fixed. The function $g(\theta)$ in (2.6) has the form

$$
g(\theta)=B\left(\frac{K_{*}-\cos \theta}{\sin \theta}\right)
$$

provided that $B \neq 0$. We shall note that if $K_{*} \neq \pm 1$, then there exists a $\theta_{*} \in(0, \pi)$ such that $g\left(\theta_{*}\right)=0$, and if $K_{*}=1$ (or $K_{*}=-1$ ), then $\lim _{\theta \rightarrow 0^{+}} g(\theta)=0\left(\right.$ or $\lim _{\theta \rightarrow \pi^{-}} g(\theta)=0$, respectively). In the following theorem, we will give lower bounds of $\lambda_{2}-\lambda_{1}$ for $K_{*} \in$ $\left[-1, \cos \frac{3 \pi}{2 n}\right] \cup\left[\cos \frac{\pi}{2 n}, 1\right]$. 
Theorem 4.1. Suppose that $(\alpha, \beta) \in \Phi_{1}$ and $(A, B)=T(\alpha, \beta)$. If $B=0$, then $A=0$ and $\lambda_{2}-\lambda_{1}=2\left(\cos \frac{\pi}{n}-\cos \frac{3 \pi}{2 n}\right)$. If $B \neq 0$, then $-1 \leq K_{*}=A / B \leq 1$. In this case,

(i) if $-1 \leq K_{*} \leq \cos \frac{3 \pi}{2 n}$, then $\lambda_{2}-\lambda_{1}>2\left(\cos \frac{\pi}{n}-\cos \frac{3 \pi}{2 n}\right)$;

(ii) if $\cos \frac{\pi}{2 n} \leq K_{*} \leq 1$, then $\lambda_{2}-\lambda_{1}>2\left(\cos \frac{\pi}{2 n}-\cos \frac{\pi}{n}\right)$.

Proof. If $B=0$, then $A=0$ by 4.2$)$. In this case, we have $g(\theta)=0$ by $(2.6), \theta_{1}=\pi /(2 n)$, and $\theta_{2}=3 \pi /(2 n)$. By using Theorem 2.1 and the monotonicity of cosine function, we have $\lambda_{2}-\lambda_{1}=2\left(\cos \frac{\pi}{n}-\cos \frac{3 \pi}{2 n}\right)$.

(i) Suppose that $B \neq 0$ and $-1 \leq K_{*} \leq \cos \frac{3 \pi}{2 n}$. There exists a $\theta_{*} \in\left[\frac{3 \pi}{2 n}, \pi\right]$ such that $g\left(\theta_{*}\right)=0$, where $g(\theta)$ is given in 4.4. By (4.3) and Lemma 3.4 , we have $g(\theta) \leq 0$ for $\theta \in\left(0, \theta_{*}\right], \cot n \theta<0$ for $\theta \in\left(\frac{\pi}{2 n}, \frac{\pi}{n}\right) \cup\left(\frac{3 \pi}{2 n}, \frac{2 \pi}{n}\right)$, and $\frac{3 \pi}{2 n} \leq \theta_{*}$. Hence

$$
\frac{\pi}{2 n}<\theta_{1}<\frac{\pi}{n} \text { and } \quad \frac{3 \pi}{2 n} \leq \theta_{2}<\frac{2 \pi}{n} .
$$

From Theorem 2.1 and the monotonicity of cosine function, we obtain that

$$
\lambda_{2}-\lambda_{1}=2 \cos \theta_{1}-2 \cos \theta_{2}>2\left(\cos \frac{\pi}{n}-\cos \frac{3 \pi}{2 n}\right) .
$$

The assertion (i) holds.

(ii) Suppose that $B \neq 0$ and $\cos \frac{\pi}{2 n} \leq K_{*} \leq 1$. There exists a $\theta_{*} \in\left[0, \frac{\pi}{2 n}\right]$ such that $g\left(\theta_{*}\right)=0$. By Lemma 3.4, $g(\theta) \geq 0$ for $\theta \in\left[\theta_{*}, \pi\right)$. Since $\cot n \theta \geq 0$ for $\theta \in$ $\left(0, \frac{\pi}{2 n}\right] \cup\left(\frac{\pi}{n}, \frac{3 \pi}{2 n}\right]$, and $\theta_{*} \leq \frac{\pi}{2 n}$. Hence

$$
0<\theta_{1} \leq \frac{\pi}{2 n} \quad \text { and } \quad \frac{\pi}{n}<\theta_{2}<\frac{3 \pi}{2 n}
$$

From Theorem 2.1, we obtain that

$$
\lambda_{2}-\lambda_{1}=2 \cos \theta_{1}-2 \cos \theta_{2}>2\left(\cos \frac{\pi}{2 n}-\cos \frac{\pi}{n}\right) .
$$

The assertion (ii) holds.

Next, we will estimate a lower bound of $\lambda_{2}-\lambda_{1}$ for $B \neq 0$ and $\cos \frac{3 \pi}{2 n}<K_{*}<\cos \frac{\pi}{2 n}$. To this end, we need the following lemma.

Lemma 4.2. Suppose that $(\alpha, \beta) \in \Phi_{1}$ and $(A, B)=T(\alpha, \beta)$. Let $\cos \frac{3 \pi}{2 n}<K_{*}<\cos \frac{\pi}{2 n}$ and $g_{B}(\theta)$ be given in (4.4), where $0<B \leq \sqrt{1+K_{*}^{2} B^{2}}$. Denote $\theta_{1}(B)<\theta_{2}(B)$ the two smallest roots of secular equation $g_{B}(\theta)=\cot n \theta$. Then

$$
\frac{1}{\sqrt{1-K_{*}^{2}}}=\underset{0<B \leq \sqrt{1+K_{*}^{2} B^{2}}}{\arg \min } \theta_{2}(B)-\theta_{1}(B) .
$$


Proof. Since $\cos \frac{3 \pi}{2 n}<K_{*}<\cos \frac{\pi}{2 n}$, there exists a $\theta_{*} \in\left(\frac{\pi}{2 n}, \frac{3 \pi}{2 n}\right)$ such that $g_{B}\left(\theta_{*}\right)=0$ for each $B$. Taking the derivative of $g_{B}(\theta)$, we have $g_{B}^{\prime}(\theta)=B\left(\frac{1-K_{*} \cos \theta}{\sin ^{2} \theta}\right)>0$ for $\theta \in(0, \pi)$. Therefore, the slope of $g_{B}(\theta)$ increases as $B$ increases. Turns out, the minimizer of $\theta_{2}(B)-\theta_{1}(B)$ takes place at a large $B$. Since $0<B \leq \sqrt{1+K_{*}^{2} B^{2}}$, the largest $B$ appears when $B=\sqrt{1+K_{*}^{2} B^{2}}$, i.e., $B=1 / \sqrt{1-K_{*}^{2}}$. This completes the proof.

Let $\cos \frac{3 \pi}{2 n}<K_{*}<\cos \frac{\pi}{2 n}$. Lemma 4.2 shows that the minimum of $\theta_{2}-\theta_{1}$ will occurs at $B=1 / \sqrt{1-K_{*}^{2}}$. Let

$$
\widehat{g}(\theta)=\frac{1}{\sqrt{1-K_{*}^{2}}}\left(\frac{K_{*}-\cos \theta}{\sin \theta}\right),
$$

and $\theta_{*}=\cos ^{-1}\left(K_{*}\right) \in\left(\frac{\pi}{2 n}, \frac{3 \pi}{2 n}\right)$. Since $\widehat{g}(\theta)$ is increasing and $\widehat{g}\left(\theta_{*}\right)=0$, we have (see Figure 4.2 for illustration)

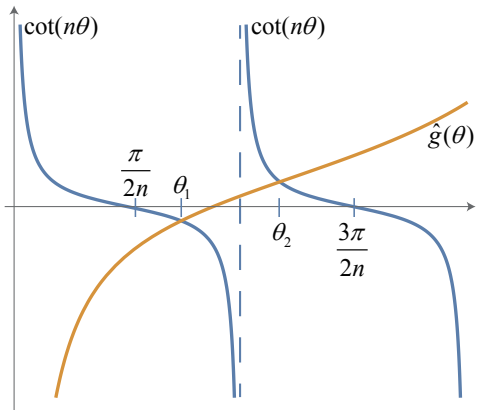

Figure 4.2: The illustration for $\cot (n \theta)$ and $\widehat{g}(\theta)$.

(i) $\frac{\pi}{2 n}<\theta_{1}<\frac{\pi}{n}<\theta_{2}<\frac{3 \pi}{2 n}$;

(ii) $\widehat{g}\left(\frac{\pi}{2 n}\right)<\widehat{g}\left(\theta_{1}\right)<0$ and $0<\widehat{g}\left(\theta_{2}\right)<\widehat{g}\left(\frac{3 \pi}{2 n}\right)$.

Then $\theta_{1}<\frac{1}{n} \cot ^{-1}\left(\widehat{g}\left(\frac{\pi}{2 n}\right)\right)<\frac{\pi}{n}<\frac{\pi}{n}+\frac{1}{n} \cot ^{-1}\left(\widehat{g}\left(\frac{3 \pi}{2 n}\right)\right)<\theta_{2}$. Hence, the lower bound of $\theta_{2}-\theta_{1}$ can be estimated as

$$
\theta_{2}-\theta_{1}>\frac{\pi}{n}+\frac{1}{n}\left[\cot ^{-1}\left(\widehat{g}\left(\frac{3 \pi}{2 n}\right)\right)-\cot ^{-1}\left(\widehat{g}\left(\frac{\pi}{2 n}\right)\right)\right] .
$$

By replacing the parameter $K_{*}$ in $\widehat{g}\left(\frac{\pi}{2 n}\right)$ and $\widehat{g}\left(\frac{3 \pi}{2 n}\right)$ by $K$ and regarding $K$ as a variable, we let $\widehat{G}_{1}(K)=\frac{1}{\sqrt{1-K^{2}}}\left(\frac{K-\cos (\pi /(2 n))}{\sin (\pi /(2 n))}\right), \widehat{G}_{2}(K)=\frac{1}{\sqrt{1-K^{2}}}\left(\frac{K-\cos (3 \pi /(2 n))}{\sin (3 \pi /(2 n))}\right)$, and

$$
\widehat{G}(K)=\cot ^{-1}\left(\widehat{G}_{2}(K)\right)-\cot ^{-1}\left(\widehat{G}_{1}(K)\right),
$$

where $K \in\left(\cos \frac{3 \pi}{2 n}, \cos \frac{\pi}{2 n}\right)$. Here we note that $\widehat{G}_{1}\left(K_{*}\right)=\widehat{g}\left(\frac{\pi}{2 n}\right)$ and $\widehat{G}_{2}\left(K_{*}\right)=\widehat{g}\left(\frac{3 \pi}{2 n}\right)$. Denote that $\theta_{1}(K)<\theta_{2}(K)$ are the two smallest roots of secular equation $B\left(\frac{K-\cos \theta}{\sin \theta}\right)=$ $\cot n \theta$. From (4.5), (4.6), and (4.7), we have

$$
\theta_{2}(K)-\theta_{1}(K)>\frac{\pi}{n}+\frac{1}{n} \widehat{G}(K) \quad \text { for each } K \in\left(\cos \frac{3 \pi}{2 n}, \cos \frac{\pi}{2 n}\right) .
$$


Then we consider the optimization problem

$$
\min _{\cos \frac{3 \pi}{2 n} \leq K \leq \cos \frac{\pi}{2 n}} \widehat{G}(K)
$$

Lemma 4.3. The minimizer of the optimization problem 4.9 is $\widehat{K}=\frac{\cos (\pi / n)}{\cos (\pi /(2 n))}$.

Proof. Taking the derivatives of $\widehat{G}_{1}(K)$ and $\widehat{G}_{2}(K)$, we have

$$
\widehat{G}_{1}^{\prime}(K)=\frac{1-K \cos (\pi /(2 n))}{\left(1-K^{2}\right)^{3 / 2} \sin (\pi /(2 n))} \quad \text { and } \quad \widehat{G}_{2}^{\prime}(K)=\frac{1-K \cos (3 \pi /(2 n))}{\left(1-K^{2}\right)^{3 / 2} \sin (3 \pi /(2 n))} .
$$

Then

$$
\begin{aligned}
\widehat{G}^{\prime}(K)= & \frac{-1}{1+\left(\widehat{G}_{2}(K)\right)^{2}} \widehat{G}_{2}^{\prime}(K)-\frac{-1}{1+\left(\widehat{G}_{1}(K)\right)^{2}} \widehat{G}_{1}^{\prime}(K) \\
= & \frac{-\sin (3 \pi /(2 n))}{\left(1-K^{2}\right) \sin ^{2}(3 \pi /(2 n))+(K-\cos (3 \pi /(2 n)))^{2}} \frac{1-K \cos (3 \pi /(2 n))}{\sqrt{1-K^{2}}} \\
& -\frac{-\sin (\pi /(2 n))}{\left(1-K^{2}\right) \sin ^{2}(\pi /(2 n))+(K-\cos (\pi /(2 n)))^{2}} \frac{1-K \cos (\pi /(2 n))}{\sqrt{1-K^{2}}} .
\end{aligned}
$$

Since

$$
\begin{gathered}
\left(1-K^{2}\right) \sin ^{2}(3 \pi /(2 n))+(K-\cos (3 \pi /(2 n)))^{2}=(1-K \cos (3 \pi /(2 n)))^{2} \\
\left(1-K^{2}\right) \sin ^{2}(\pi /(2 n))+(K-\cos (\pi /(2 n)))^{2}=(1-K \cos (\pi /(2 n)))^{2}
\end{gathered}
$$

we have

$$
\widehat{G}^{\prime}(K)=\frac{\sin (\pi /(2 n))-\sin (3 \pi /(2 n))+K \sin (\pi / n)}{\sqrt{1-K^{2}}(1-K \cos (3 \pi /(2 n)))(1-K \cos (\pi /(2 n)))} .
$$

Hence, $\widehat{G}^{\prime}(K)=0$ if and only if $\sin (\pi /(2 n))-\sin (3 \pi /(2 n))+K \sin (\pi / n)=0$. This implies that the critical point

$$
\widehat{K}=\frac{\sin (3 \pi /(2 n))-\sin (\pi /(2 n))}{\sin (\pi / n)}=\frac{2 \cos (\pi / n) \sin (\pi /(2 n))}{2 \cos (\pi /(2 n)) \sin (\pi /(2 n))}=\frac{\cos (\pi / n)}{\cos (\pi /(2 n))} .
$$

Next, we claim that $\cos \frac{3 \pi}{2 n}<\widehat{K}<\cos \frac{\pi}{2 n}$. Since $\cos ^{2} \frac{\pi}{2 n}=\cos \frac{\pi}{n}+\sin ^{2} \frac{\pi}{2 n}>\cos \frac{\pi}{n}$, we have $\widehat{K}<\cos \frac{\pi}{2 n}$. Using the fact that $\cos \frac{3 \pi}{2 n}<\cos \frac{\pi}{n}<\widehat{K}$, we have $\cos \frac{3 \pi}{2 n}<\widehat{K}<\cos \frac{\pi}{2 n}$.

From 4.10, it is easily seen that if $\cos \frac{3 \pi}{2 n}<K<\widehat{K}$ then $\widehat{G}^{\prime}(K)<0$, and if $\widehat{K}<$ $K<\cos \frac{\pi}{2 n}$ then $\widehat{G}^{\prime}(K)>0$. Hence, $\widehat{K}=\frac{\cos (\pi / n)}{\cos (\pi /(2 n))}$ is the minimizer of the optimization problem 4.9.

Combining the previous lemmas, we have the following result. 
Theorem 4.4. Suppose that $(\alpha, \beta) \in \Phi_{1}$, where $\Phi_{1}$ is given in 4.1 . Let $(A, B)=T(\alpha, \beta)$, $B \neq 0$, and $K_{*}=A / B$. If $\cos \frac{3 \pi}{2 n}<K_{*}<\cos \frac{\pi}{2 n}$, then

$$
\lambda_{2}-\lambda_{1}>\frac{4}{n} \sin \left(\frac{\pi}{2 n}\right) \cos ^{-1}\left(\frac{1}{2} \sec \frac{\pi}{2 n}\right) .
$$

Proof. By Lemma 4.3 we let $\widehat{K}=\frac{\cos (\pi / n)}{\cos (\pi /(2 n))}$. Then $\widehat{G}_{1}(\widehat{K})=\frac{\cos (\pi / n)-\cos ^{2}(\pi /(2 n))}{\sin (\pi /(2 n)) \sqrt{\cos ^{2}(\pi /(2 n))-\cos ^{2}(\pi / n)}}$. Since $\cos (\pi / n)-\cos ^{2}(\pi /(2 n))=-\sin ^{2}(\pi /(2 n))$ and

$$
\cos ^{2}(\pi /(2 n))-\cos ^{2}(\pi / n)=\left(4 \cos ^{2}(\pi /(2 n))-1\right) \sin ^{2}(\pi /(2 n)),
$$

we have $\widehat{G}_{1}(\widehat{K})=\frac{-1}{\sqrt{4 \cos ^{2}(\pi /(2 n))-1}}$. Similarly, $\widehat{G}_{2}(\widehat{K})=\frac{\cos (\pi / n)-\cos (\pi /(2 n)) \cos (3 \pi /(2 n))}{\sin (3 \pi /(2 n)) \sqrt{\cos ^{2}(\pi /(2 n))-\cos ^{2}(\pi / n)}}$. Since $\cos (\pi / n)-\cos (\pi /(2 n)) \cos (3 \pi /(2 n))=\sin (\pi /(2 n)) \sin (3 \pi /(2 n))$, it follows from 4.11 that $\widehat{G}_{2}(\widehat{K})=\frac{1}{\sqrt{4 \cos ^{2}(\pi /(2 n))-1}}$.

Since $\widehat{G}_{1}(\widehat{K})=-\widehat{G}_{2}(\widehat{K})<0$, we have $\cot ^{-1}\left(\widehat{G}_{1}(\widehat{K})\right)=\pi-\cot ^{-1}\left(\widehat{G}_{2}(\widehat{K})\right)$. It follows from (4.7) and 4.8 that for $K_{*} \in\left(\cos \frac{3 \pi}{2 n}, \cos \frac{\pi}{2 n}\right)$,

$$
\theta_{2}\left(K_{*}\right)-\theta_{1}\left(K_{*}\right)>\frac{2}{n} \cot ^{-1}\left(\frac{1}{\sqrt{4 \cos ^{2}(\pi /(2 n))-1}}\right)=\frac{2}{n} \cos ^{-1}\left(\frac{1}{2} \sec \frac{\pi}{2 n}\right) .
$$

Therefore, there exists a $\theta \in\left(\theta_{1}\left(K_{*}\right), \theta_{2}\left(K_{*}\right)\right)$ such that

$$
\lambda_{2}-\lambda_{1}=2 \sin \theta\left(\theta_{2}\left(K_{*}\right)-\theta_{1}\left(K_{*}\right)\right)>\frac{4}{n} \sin \theta \cos ^{-1}\left(\frac{1}{2} \sec \frac{\pi}{2 n}\right) .
$$

Since $\frac{\pi}{2 n} \leq \theta_{1}\left(K_{*}\right)$, we have

$$
\lambda_{2}-\lambda_{1}>\frac{4}{n} \sin \left(\frac{\pi}{2 n}\right) \cos ^{-1}\left(\frac{1}{2} \sec \frac{\pi}{2 n}\right) .
$$

- For the case $(\alpha, \beta) \in \Phi_{2}=\left\{(\alpha, \beta) \in \mathbb{R}^{2} \mid \alpha<-1, \beta \leq 1\right.$ and $\left.\alpha \beta<1\right\}$ :

Suppose that $(\alpha, \beta) \in \Phi_{2}$ and $(A, B)=T(\alpha, \beta)$. Then

$$
(A, B) \in T\left(\Phi_{2}\right)=\left\{(A, B) \in \mathbb{R}^{2} \mid-1<B<-A \text { and } A \leq B\right\}
$$

as shown in 4.2). From Theorem 3.6, we see that $0<\lambda_{1}<\mu_{1}<\lambda_{2}<\mu_{2}$, where $\mu_{1}$ and $\mu_{2}$ are given in 1.4. From Figure 4.1, the region $T\left(\Phi_{2}\right)$ can be written as

$$
\begin{aligned}
T\left(\Phi_{2}\right)= & \{(K B, B) \mid K \geq 1 \text { and }-1<B<0\} \cup\{(A, 0) \mid A<0\} \\
& \cup\{(K B, B) \mid K<-1 \text { and } 0<B\} .
\end{aligned}
$$

Assume that $B \neq 0$. Then there is a $K_{*}$ with $K_{*} \geq 1$ such that $A=K_{*} B$ and $-1<B<0$, or there is a $K_{*}$ with $K_{*}<-1$ such that $A=K_{*} B$ and $0<B$. Let $g(\theta)$ be given in (4.4). 
In both cases, $g(\theta)<0$ for all $\theta \in(0, \pi)$. Since $\cot n \theta<0$ for $\theta \in\left(\frac{\pi}{2 n}, \frac{\pi}{n}\right) \cup\left(\frac{3 \pi}{2 n}, \frac{2 \pi}{n}\right)$, we have

$$
\frac{\pi}{2 n}<\theta_{1}<\frac{\pi}{n} \text { and } \quad \frac{3 \pi}{2 n} \leq \theta_{2}<\frac{2 \pi}{n} .
$$

Assume that $B=0$. Then $g(\theta)=A \csc \theta$. Since $A<0$, we have $g(\theta)<0$ for all $\theta \in(0, \pi)$. Hence, the two smallest roots of the secular equation $g(\theta)=\cot n \theta$ also satisfy 4.12 . From Theorem 2.1, we have the following result.

Theorem 4.5. Suppose that $(\alpha, \beta) \in \Phi_{2}$, where $\Phi_{2}$ is given in (4.1). Then

$$
\lambda_{2}-\lambda_{1}=2 \cos \theta_{1}-2 \cos \theta_{2}>2\left(\cos \frac{\pi}{n}-\cos \frac{3 \pi}{2 n}\right) .
$$

- For the case $(\alpha, \beta) \in \Phi_{3}=\left\{(\alpha, \beta) \in \mathbb{R}^{2} \mid \beta>1\right.$ and $\left.\alpha \beta<1\right\}$ :

Suppose that $(\alpha, \beta) \in \Phi_{3}$ and $(A, B)=T(\alpha, \beta)$. Here,

$$
\begin{aligned}
T\left(\Phi_{3}\right)= & \left\{(A, B) \in \mathbb{R}^{2} \mid-1<B \leq A-1 / n\right\} \\
& \cup\left\{(A, B) \in \mathbb{R}^{2} \mid-1<B<A<B+1 / n\right\}
\end{aligned}
$$

which shows in 4.2 (see Figure 4.1 for illustration). Then we have the following result.

Theorem 4.6. Suppose that $(\alpha, \beta) \in \Phi_{3}$ and $(A, B)=T(\alpha, \beta)$, where $\Phi_{3}$ is given in (4.1). Then $B>-1$ and

(i) if $B \leq A-1 / n$, then $\lambda_{2}-\lambda_{1}>2-2 \cos \frac{\pi}{n}$;

(ii) if $B<A<B+1 / n$, then $\lambda_{2}-\lambda_{1}>2\left(\cos \frac{\pi}{2 n}-\cos \frac{\pi}{n}\right)$.

Proof. Suppose that $(\alpha, \beta) \in \Phi_{3}$ and $(A, B)=T(\alpha, \beta)$. From $(4.2)$, we have $-1<B<A$. Suppose that $B \leq A-1 / n$, it follows from Theorem 3.6 that $\lambda_{1} \leq 0<\mu_{1}<\lambda_{2}$. Hence, $\lambda_{2}-\lambda_{1}>2-2 \cos \frac{\pi}{n}$. Then assertion (i) holds.

For the case $B<A<B+1 / n$, let $\widehat{A}=\widehat{B}=B$. Then

$$
g(\theta)=\frac{A-B \cos \theta}{\sin \theta}=\widehat{g}(\theta)+\frac{A-\widehat{A}}{\sin \theta},
$$

where $\widehat{g}(\theta)=\frac{\widehat{A}-\widehat{B} \cos \theta}{\sin \theta}$ and $A-\widehat{A}>0$. Note that $\frac{A-\widehat{A}}{\sin \theta}>0$ for all $\theta \in(0, \pi)$.

(a) If $B \geq 0$, then $\widehat{g}(\theta) \geq 0$ for $\theta \in(0, \pi)$. From 4.13), we obtain that $0<\theta_{1}<\frac{\pi}{2 n}<$ $\frac{\pi}{n}<\theta_{2}$. Hence, $\lambda_{2}-\lambda_{1}>2\left(\cos \frac{\pi}{2 n}-\cos \frac{\pi}{n}\right)$.

(b) If $B<0$, then $\widehat{g}(\theta)<0$ and decreasing on $\theta \in(0, \pi)$. Since $\frac{A-\widehat{A}}{\sin \theta}>0$ and decreasing on $\theta \in(0, \pi / 2)$, from 4.13$)$ we obtain $g(\theta)$ is also decreasing on $\theta \in(0, \pi / 2)$. It follows from Theorem 3.6 that $0<\theta_{1}<\frac{\pi}{n}<\theta_{2}<\frac{2 \pi}{n}$ and $\theta_{2}-\theta_{1}>\pi / n$. Then we have $\lambda_{2}-\lambda_{1}=2\left(\cos \theta_{1}-\cos \theta_{2}\right)>2-2 \cos \frac{\pi}{n}$.

This completes the proof. 
In this section, we first partition the parameter domain $\Omega_{1}$ into three regions. We also estimate the first eigenvalue gaps for these 3 regions in Theorems 4.1, 4.4, 4.5, and 4.6. We summarize a result of the global gap of the first two eigenvalues for $(\alpha, \beta) \in \Omega_{1}$ in the following remark.

Remark 4.7. For each positive integer $n \geq 2$, we have

$$
\cos \frac{\pi}{n}-\cos \frac{3 \pi}{2 n}>1-\cos \frac{\pi}{n}>\cos \frac{\pi}{2 n}-\cos \frac{\pi}{n} .
$$

(i) Using the Mean Value Theorem, we obtain that $\cos \frac{\pi}{2 n}-\cos \frac{\pi}{n} \geq \frac{\pi}{2 n} \sin \frac{\pi}{2 n}$. Due to the monotonicity of $\cos ^{-1}$ and sec and $n \geq 2$ a calculation yields

$$
\cos ^{-1}\left(\frac{1}{2} \sec \frac{\pi}{2 n}\right) \leq \cos ^{-1}\left(\frac{1}{2} \sec \frac{\pi}{4}\right)=\frac{\pi}{4}
$$

Hence, $\frac{4}{n} \sin \left(\frac{\pi}{2 n}\right) \cos ^{-1}\left(\frac{1}{2} \sec \frac{\pi}{2 n}\right) \leq \frac{\pi}{n} \sin \left(\frac{\pi}{2 n}\right) \leq 2\left(\cos \frac{\pi}{2 n}-\cos \frac{\pi}{n}\right)$. It follows from 4.14 and Theorems 4.1, 4.4, 4.5, and 4.6 that for each $(\alpha, \beta) \in \Omega_{1}$,

$$
\lambda_{2}(\alpha, \beta)-\lambda_{1}(\alpha, \beta)>\frac{4}{n} \sin \left(\frac{\pi}{2 n}\right) \cos ^{-1}\left(\frac{1}{2} \sec \frac{\pi}{2 n}\right) .
$$

This proves the the inequality in (1.5).

(ii) Taylor series approximation gives

$$
\begin{aligned}
& \frac{4}{n} \sin \left(\frac{\pi}{2 n}\right) \cos ^{-1}\left(\frac{1}{2} \sec \frac{\pi}{2 n}\right) \\
= & \frac{4}{n}\left(\frac{\pi}{2 n}-\frac{1}{3 !}\left(\frac{\pi}{2 n}\right)^{3}+\cdots\right) \cos ^{-1}\left(\frac{1}{2}+\frac{1}{2}\left(\frac{\pi}{2 n}\right)^{2}+\cdots\right) \\
= & \frac{2 \pi^{2}}{3 n^{2}}+O\left(\frac{1}{n^{4}}\right) .
\end{aligned}
$$

This proves the equality in 1.5 .

\section{The monotonicity property of eigenvalues}

The monotonicity property of eigenvalues can be investigated by using the secular equation $g(\theta)=\cot n \theta$. In this section, we consider the parameters $(\alpha, \beta) \in \Phi_{1}$ which is defined in 4.1). In this parameter region, it follows from (4.3) that the pair $(A, B) \in T\left(\Phi_{1}\right)$ satisfies $B \neq 0$ and $-1 \leq K_{*}=A / B \leq 1$. Later, we shall fix the ratio $K_{*}$ and consider a parameter curve $\left\{(\alpha, \beta) \in \Phi_{1} \mid K_{*}=A(\alpha, \beta) / B(\alpha, \beta)\right\}$. Along this parameter curve, we have the following monotonicity properties.

Theorem 5.1. Let $(\alpha, \beta) \in \Phi_{1} \backslash\{(-1,1)\}$ and $(A, B)=T(\alpha, \beta)$. Therefore, $B \neq 0$ and $-1 \leq K_{*}=A / B \leq 1$. Denote $\widehat{\lambda}_{1}<\widehat{\lambda}_{2}<\cdots<\widehat{\lambda}_{n}$ the eigenvalues of $L(\widehat{\alpha}, \widehat{\beta})$. 
(i) Suppose that $-1<K_{*}<1$ and $k_{*}$ is an integer such that

$$
\cos \frac{\left(2 k_{*}+1\right) \pi}{2 n}<K_{*}<\cos \frac{\left(2 k_{*}-1\right) \pi}{2 n} .
$$

If $\alpha<\widehat{\alpha}<1$ and $\widehat{\beta}=\left(K_{*}-\widehat{\alpha}\right) /\left(1-K_{*} \widehat{\alpha}\right)$ with $\widehat{\alpha}<\widehat{\beta}$, then $(\widehat{\alpha}, \widehat{\beta}) \in \Phi_{1}$ and

$$
0<\lambda_{1}<\widehat{\lambda}_{1}<\lambda_{2}<\widehat{\lambda}_{2}<\cdots<\lambda_{k_{*}}<\widehat{\lambda}_{k_{*}}<\widehat{\lambda}_{k_{*}+1}<\lambda_{k_{*}+1}<\cdots<\widehat{\lambda}_{n}<\lambda_{n}<4 .
$$

(ii) Suppose that $K_{*}=1$. We have $-1<\alpha<1$ and $\beta=1$. If $\alpha<\widehat{\alpha}$ and $\widehat{\beta}=1$, then

$$
0<\widehat{\lambda}_{1}<\lambda_{1}<\widehat{\lambda}_{2}<\lambda_{2}<\cdots<\widehat{\lambda}_{n}<\lambda_{n}<4 \text {. }
$$

(iii) Suppose that $K_{*}=-1$. We have $\alpha=-1$ and $-1<\beta<1$. If $\widehat{\alpha}=-1$ and $\beta<\widehat{\beta}$, then

$$
0<\widehat{\lambda}_{1}<\lambda_{1}<\widehat{\lambda}_{2}<\lambda_{2}<\cdots<\widehat{\lambda}_{n}<\lambda_{n}<4 \text {. }
$$

Proof. Let $(\alpha, \beta) \in \Phi_{1} \backslash\{(-1,1)\}$. From 4.2 , we have $B \neq 0$ and $-1 \leq K_{*}=A / B \leq 1$.

(i) Suppose that $-1<K_{*}<1, \alpha<\widehat{\alpha}<1$, and $\widehat{\beta}=\left(K_{*}-\widehat{\alpha}\right) /\left(1-K_{*} \widehat{\alpha}\right)$ with $\widehat{\alpha}<\widehat{\beta}$. Then we have

$$
1-K_{*} \widehat{\alpha}>0 \quad \text { and } \quad K_{*}-2 \widehat{\alpha}+K_{*} \widehat{\alpha}^{2}>0
$$

Since $\left(1-K_{*} \widehat{\alpha}\right)-\left(K_{*}-\widehat{\alpha}\right)=\left(1-K_{*}\right)(1+\widehat{\alpha})>0$, we have $\widehat{\beta}<1$. Hence, $(\widehat{\alpha}, \widehat{\beta}) \in \Phi_{1}$.

By substituting $\widehat{\beta}=\left(K_{*}-\widehat{\alpha}\right) /\left(1-K_{*} \widehat{\alpha}\right)$ into $(3.2)$, we have $A(\widehat{\alpha}, \widehat{\beta}) / B(\widehat{\alpha}, \widehat{\beta})=(\widehat{\alpha}+$ $\widehat{\beta}) /(1+\widehat{\alpha} \widehat{\beta})=\left(\widehat{\alpha}-K_{*} \widehat{\alpha}^{2}+K_{*}-\widehat{\alpha}\right) /\left(1-K_{*} \widehat{\alpha}+K_{*} \widehat{\alpha}-\widehat{\alpha}^{2}\right)=K_{*}$ and $B(\widehat{\alpha}) \equiv B(\widehat{\alpha}, \widehat{\beta})=$ $\left(1-\widehat{\alpha}^{2}\right) /\left(1-2 K_{*} \widehat{\alpha}+\widehat{\alpha}^{2}\right)$. It follows from $(5.2)$ that

$$
B^{\prime}(\widehat{\alpha})=\frac{2 K_{*} \widehat{\alpha}^{2}-4 \widehat{\alpha}+2 K_{*}}{\left(1-2 K_{*} \widehat{\alpha}+\widehat{\alpha}^{2}\right)^{2}}>0 .
$$

This implies that $B(\widehat{\alpha})$ is increasing in $\widehat{\alpha}$. Hence, $B(\alpha)<B(\widehat{\alpha})$ because $\alpha<\widehat{\alpha}$.

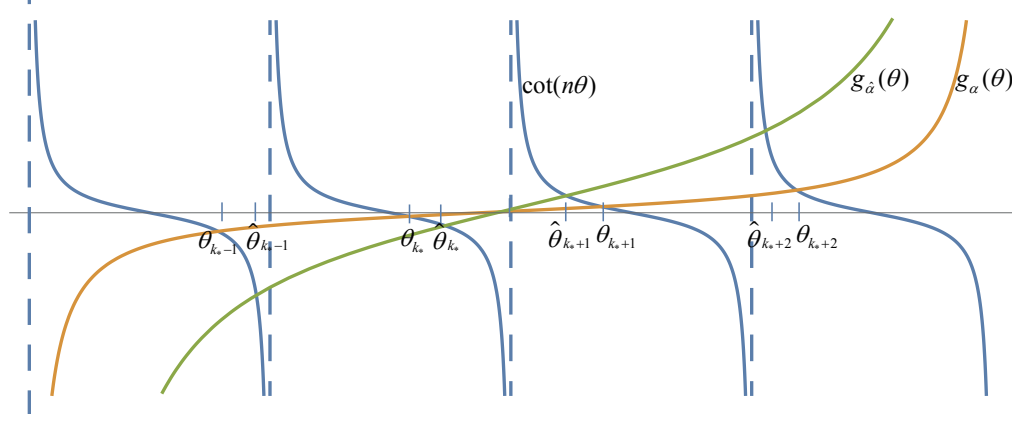

Figure 5.1: The illustration for Theorem 5.1 . 
Let $g_{\widehat{\alpha}}(\theta)=B(\widehat{\alpha})\left(\frac{K_{*}-\cos \theta}{\sin \theta}\right)$. Since $A(\widehat{\alpha}, \widehat{\beta}) / B(\widehat{\alpha}, \widehat{\beta})=K_{*}$, we obtain that $g_{\widehat{\alpha}}(\theta)=$ $\cot n \theta$ is the secular equation of $(1.3)$ with $(\alpha, \beta)=\left(\widehat{\alpha},\left(K_{*}-\widehat{\alpha}\right) /\left(1-K_{*} \widehat{\alpha}\right)\right)$. Suppose that $0<\theta_{1}<\theta_{2}<\cdots<\theta_{n}<\pi$ and $0<\widehat{\theta}_{1}<\widehat{\theta}_{2}<\cdots<\widehat{\theta}_{n}<\pi$ are the roots of $g_{\alpha}(\theta)=\cot n \theta$ and $g_{\widehat{\alpha}}(\theta)=\cot n \theta$, respectively. Then we have $\lambda_{j}=2-2 \cos \theta_{j}$ and $\widehat{\lambda}_{j}=2-2 \cos \widehat{\theta}_{j}$ for $j=1, \ldots, n$. It follows from (5.1) that $g_{\widehat{\alpha}}(\theta)=0$ has solution $\theta_{*}$ with $\widehat{\theta}_{k_{*}}<\theta_{*}<\widehat{\theta}_{k_{*}+1}$. Since $B(\alpha)<B(\widehat{\alpha})$, we have (see Figure 5.1 for illustration)

$$
\theta_{1}<\widehat{\theta}_{1}<\theta_{2}<\widehat{\theta}_{2}<\cdots<\theta_{k_{*}}<\widehat{\theta}_{k_{*}}<\widehat{\theta}_{k_{*}+1}<\theta_{k_{*}+1}<\cdots<\widehat{\theta}_{n}<\theta_{n}
$$

Hence, the assertion (i) holds.

(ii) Let $K_{*}=(\alpha+\beta) /(1+\alpha \beta)=1$, then $0=1+\alpha \beta-\alpha-\beta=(\alpha-1)(\beta-1)$. Since $-1 \leq \alpha \leq \beta \leq 1$ and $\alpha \beta<1$, we have $-1<\alpha<1$ and $\beta=1$. Suppose that $\alpha<\widehat{\alpha}$ and $\widehat{\beta}=1$, then $A(\widehat{\alpha}, \widehat{\beta}) / B(\widehat{\alpha}, \widehat{\beta})=(\widehat{\alpha}+\widehat{\beta}) /(1+\widehat{\alpha} \widehat{\beta})=1=K_{*}$ and $B(\widehat{\alpha}) \equiv B(\widehat{\alpha}, \widehat{\beta})=$ $(1+\widehat{\alpha}) /(1-\widehat{\alpha})$. It is easy to see that $B(\widehat{\alpha})$ is increasing in $\widehat{\alpha}$. Let $g_{\widehat{\alpha}}(\theta)=B(\widehat{\alpha})\left(\frac{1-\cos \theta}{\sin \theta}\right)$. Then $\lim _{\theta \rightarrow 0^{+}} g_{\widehat{\alpha}}(\theta)=0, g_{\widehat{\alpha}}(\theta)$ is increasing in $\theta$. Let $0<\theta_{1}<\theta_{2}<\cdots<\theta_{n}<\pi$ and $0<\widehat{\theta}_{1}<\widehat{\theta}_{2}<\cdots<\widehat{\theta}_{n}<\pi$ are the roots of $g_{\alpha}(\theta)=\cot n \theta$ and $g_{\widehat{\alpha}}(\theta)=\cot n \theta$, respectively. Then we have $\widehat{\theta}_{1}<\theta_{1}<\widehat{\theta}_{2}<\theta_{2}<\cdots<\widehat{\theta}_{n}<\theta_{n}$. Hence, the assertion (ii) holds.

Similarly, we can show that the assertion (iii) holds.

\section{References}

[1] M. A. Al-Gwaiz, Sturm-Liouville Theory and its Applications, Springer Undergraduate Mathematics Series, Springer-Verlag London, London, 2008.

[2] M. S. Ashbaugh and R. D. Benguria, Optimal bounds for ratios of eigenvalues of onedimensional Schrödinger operators with Dirichlet boundary conditions and positive potentials, Comm. Math. Phys. 124 (1989), no. 3, 403-415.

[3] _ Some eigenvalue inequalities for a class of Jacobi matrices, Linear Algebra Appl. 136 (1990), 215-234.

[4] _ Eigenvalue ratios for Sturm-Liouville operators, J. Differential Equations 103 (1993), no. 1, 205-219.

[5] F. Chung and S.-T. Yau, Discrete Green's functions, J. Combin. Theory Ser. A 91 (2000), no. 1-2, 191-214.

[6] R. L. Frank and L. Geisinger, Semi-classical analysis of the Laplace operator with Robin boundary conditions, Bull. Math. Sci. 2 (2012), no. 2, 281-319. 
[7] R. A. Horn and C. R. Johnson, Matrix Analysis, Cambridge University Press, Cambridge, 1990 .

[8] Y. L. Huang and C. K. Law, Eigenvalue ratios for the regular Sturm-Liouville system, Proc. Amer. Math. Soc. 124 (1996), no. 5, 1427-1436.

[9] M. Jarret and S. P. Jordan, The fundamental gap for a class of Schrödinger operators on path and hypercube graphs, J. Math. Phys. 55 (2014), no. 5, 052104, 16 pp.

[10] J. Juang, W.-W. Lin and S.-F. Shieh, Eigenvalue problems for one-dimensional discrete Schrödinger operators with symmetric boundary conditions, SIAM J. Matrix Anal. Appl. 23 (2001), no. 2, 524-533.

[11] A. Khalkhuzhaev, S. Y. Kholmatov and M. Pardabaev, Expansion of eigenvalues of rank-one perturbations of the discrete bilaplacian, arXiv:1910.01369.

[12] E. L. Korotyaev and J. S. Møller, Weighted estimates for the Laplacian on the cubic lattice, Ark. Mat. 57 (2019), no. 2, 397-428.

[13] E. Korotyaev and N. Saburova, Spectral estimates for the Schrödinger operator on periodic discrete graphs, St. Petersburg Math. J. 30 (2019), no. 4, 667-698.

[14] K. Liu and H. Xu, Open problems, Notices of the International Congress of Chinese Mathematicians 7 (2019), no. 2, 72-73.

[15] M. A. Lyalinov, Comments on the eigenfunctions and eigenvalues of the Laplace operator in an angle with Robin boundary conditions, J. Math. Sci. (N.Y.) 252 (2021), no. 5, 646-653.

[16] I. M. Singer, B. Wong, S.-T. Yau and S. S.-T. Yau, An estimate of the gap of the first two eigenvalues in the Schrödinger operator, Ann. Scuola Norm. Sup. Pisa Cl. Sci. (4) 12 (1985), no. 2, 319-333.

[17] R. S. Varga, Matrix Iterative Analysis, Second revised and expanded edition, Springer Series in Computational Mathematics 27, Springer-Verlag, Berlin, 2000.

[18] A. Zettl, Sturm-Liouville Theory, Mathematical Surveys and Monographs 121, American Mathematical Society, Providence, RI, 2005.

[19] Q. Zhu, Z. Zhou and L. Wang, Existence and stability of discrete solitons in nonlinear Schrödinger lattices with hard potentials, Phys. D 403 (2020), 132326, 10 pp. 


\section{Yueh-Cheng Kuo}

Department of Applied Mathematics, National University of Kaohsiung, Kaohsiung 811, Taiwan

E-mail address: yckuo@nuk.edu.tw

Shih-Feng Shieh

Department of Mathematics, National Taiwan Normal University, Taipei 116, Taiwan

E-mail address: sf shieh@ntnu.edu.tw 